\title{
Ensinando/Praticando Mindfulness por meio do Pensamento Computacional para Alunos do Ensino Fundamental
}

\author{
Felipe Faustino de Souza ${ }^{1}$, Maria Augusta S. N. Nunes ${ }^{2,3}$ \\ ${ }^{1}$ Mestrando do Programa de Pós-Graduação em Ciência da Computação - Universidade \\ Federal do Sergipe (UFS) \\ ${ }^{2}$ Professora do Programa de Pós-Graduação em Ciência da Computação - Universidade \\ Federal do Sergipe (UFS) \\ Av. Marechal Rondon, s/n - São Cristóvão - SE - Brasil \\ ${ }^{3}$ Professora do Programa de Pós-Graduação em Informática - Universidade Federal do \\ Estado do Rio de Janeiro (UNIRIO) \\ Av. Pasteur, 296 - Urca, Rio de Janeiro - RJ - Brasil \\ \{felipe.faustino,gutanunes\}@gmail.com

\begin{abstract}
Mindfulness practices have been applied in several areas, including education, to mitigate the lack of attention and positively impacting students' social and emotional development. This innovative work aims the elaboration and validation of a guide of Educational Activities that helps the teaching of mindfulness trimmed by methodological resources of Computational Thinking (CT) for elementary school students in subjects of Physical Education and Art. It is believed that this application contributes to the corporeal and spatial development, creativity and skills related to the four pillars of the CT and the five skills of the 21st century (\#5c21).
\end{abstract}

Resumo. Práticas de mindfulness (atenção plena) tem sido propostas em diversas áreas, inclusive na educação, com o intuito de mitigar a falta de atenção e impactando positivamente no desenvolvimento social e emocional dos alunos. Esse trabalho tem como objetivo inovador elaborar e validar um Guia de Atividades Pedagógicas Desplugadas que auxilie o ensino/prática de mindfulness amparado por recursos metodológicos do Pensamento Computacional (PC) para alunos do ensino fundamental nas disciplinas de Educação Física e Arte. Acredita-se que essa aplicação contribua para o desenvolvimento corporal e espacial, da criatividade e das habilidades relacionadas aos 4 pilares do PC e as 5 competências do século 21 (\#5c21).

\section{Introdução}

O sucesso no desempenho escolar está estreitamente conectado com a qualidade do ambiente escolar, ambiente aquele sem distrações e com a presença da atenção plena no material de estudo. Em paralelo, professores relatam a falta de atenção em sala de aula e a relacionam com a dificuldade de aprendizagem propiciando dificuldades no iniciar, organizar e finalizar de tarefa, tornando assim o processo de aprendizagem de conceitos e habilidades mais custoso [Ramos et al., 2018][Smith and Strick, 2009][Rahal, 2018][Black and Fernando, 2014].

Posto este cenário, o desafio proposto aos professores se torna imenso tendo em vista que a eles competem a manutenção da atenção e as especificidades da aprendizagem de cada aluno [Black and Fernando, 2014]. Como forma mitigar a falta de atenção em 
VIII Congresso Brasileiro de Informática na Educação (CBIE 2019)

Anais dos Workshops do VIII Congresso Brasileiro de Informática na Educação (WCBIE 2019)

sala de aula a literatura apresenta diversas intervenções como: gamification [Ramos et al., 2018], atividades motivacionais [Gregory and Kaufeldt, 2015], computer-based intervention [Rabiner et al., 2010], práticas de mindfulness [Napoli et al., 2005], entre outras.

A prática de mindfulness aponta melhorias não somente na atenção, mas também no desenvolvimento social e emocional da criança e do adolescente, reduzindo assim níveis de estresse e ansiedade, propiciando um melhor desempenho em sala de aula advindos da capacidade de se manterem atentos e gerenciarem o estresse e outros sentimentos difíceis proporcionando ao aluno um estado pleno de consciência de quem é e de onde está (consciência corporal e espacial) [Napoli et al., 2005][Burke, 2010]. Ajudando assim ao aluno avaliar suas metas e encontrar a melhor forma de agir para atingi-las [Williams and Penman, 2015].

Zenner et al. [2014] adicionam que essa qualidade de atenção plena proporciona o desenvolvimento de ética, criatividade e a habilidade de resolver problemas e Karunananda et al. [2016] complementam que a prática de mindfulness estimula o desenvolvimento de habilidades cognitivas, o pensamento e a solução de problemas.

Não somente por meio de meditação, o estado de mindfulness informal, estado de consciência, pode ser aplicado em atividades diárias como: caminhar, escovar os dentes, respirar, interagir com pessoas, realizar atividades escolares, etc. O que difere é posicionamento voltando a sua atenção para o momento presente, sem julgar ou racionalizar o que acontece. Permitindo assim a vivência de experiências agindo com criatividade em situações do cotidiano [Alves, 2018][Simón, 2007][Black and Fernando, 2014][Ager et al. 2015].

Santos et al. [2019] afirmam que a criatividade, a produtividade e a inventividade são algumas das habilidades estimuladas com o desenvolvimento dos pilares do Pensamento Computacional (PC) (Decomposição, Reconhecimento de Padrões, Abstração e Algoritmo) [Brackmann, 2016]. Além da criatividade, proposta por Romero [2016] e [Romero at el. 2019] adicionam como habilidades-chave para o século 21 (\#5c21) o pensamento crítico, colaboração, resolução de problemas e o próprio PC. Santos et al. [2019] adicionam o desenvolvimento do pensamento algoritmo, aprendizagem colaborativa, resolução de problemas, raciocínio lógico e interpretação textual como habilidades oriundas da aplicação dos quatro pilares do PC.

Além desses estímulos a criatividade/co-criatividade, bem como o PC, podem ser desenvolvidos de forma interdisciplinar onde, em seu trabalho, Santos [2019] possibilitou o desenvolvimento do PC Desplugado aplicado às disciplinas de Língua Portuguesa e Matemática, assim, alcançando um resultado positivo, caracterizando o PC como intermediador da Ciência da Computação (CC) e as mais diversas áreas [Wing, 2006].

\subsection{Motivação}

A falta de atenção generalizada impacta no processo de aprendizagem refletindo no baixo desempenho aferido pelo PISA (Programme for International Student Assessment), adicionados a pouca ou nenhuma infraestrutura em escolas públicas e ainda a preferência por parte dos docentes em atividades Desplugadas [Rodrigues et al., 2018].

Reforçando a falta de estudos que elucidem o ensino/prática de mindfulness em conjunto aos conceitos de PC apontando assim a necessidade de criação de artefatos e 
VIII Congresso Brasileiro de Informática na Educação (CBIE 2019)

Anais dos Workshops do VIII Congresso Brasileiro de Informática na Educação (WCBIE 2019)

estratégias de ensino de baixo custo que contemplem esse cenário e a popularização, por conseguinte, da CC.

\subsection{Problema}

Considerado a realidade socioeconômica das escolas brasileiras, o baixo índice de desempenho escolar e a falta de atenção observada pelos professores, o problema que essa dissertação pretende resolver é apresentado pela questão de pesquisa seguinte:

"Como o ensino de mindfulness por meio do PC de forma Desplugada e interdisciplinar pode auxiliar na melhoria da atenção, consequentemente da consciência (corporal e espacial), criatividade e desempenho escolar de crianças nas disciplinas de Educação Física e Arte e, consequentemente, compartilhem o eixo temático Dança (Movimento corporal, Organização interna e Relações entre as partes do corpo e o todo na construção do movimento) no ensino e aprendizagem de estudantes do ensino fundamental?"

\subsection{Objetivos}

O objetivo desta dissertação é elaborar, experimentar e validar um Guia de Atividades Pedagógicas Desplugadas que auxilie o ensino/prática de mindfulness usando como ferramenta o Pensamento Computacional para alunos do ensino fundamental nas disciplinas de Educação Física e Arte.

Tomando como ponto de partida o objetivo principal, segue os objetivos específicos: Redigir três Mapeamento Sistemático da literatura. Os dois primeiros sobre mindfulness e PC Desplugado aplicados à educação básica de forma separada, e o último de forma conjunta, assim elucidando as estratégias aplicadas e os artefatos utilizados; coletar dados através de Survey com professores das disciplinas em questão; Confeccionar Artefatos Desplugados; elaborar um Guia de Ensino de mindfulness por meio do PC; experimentar o Guia de Ensino de mindfulness por meio do PC.

\section{Metodologia de Pesquisa}

Para atingir os objetivos desta dissertação serão cumpridas 5 etapas, as quais serão aplicadas em uma escola da rede pública ensino da cidade de Aracaju - Sergipe/SE, com alunos do $6^{\mathrm{a}}$ ano do ensino fundamental (alunos entre 10 e 11 anos) nas disciplinas de Educação Física e Arte.

A Primeira Etapa será caracterizada pela a realização da Pesquisa Bibliográfica. A Segunda Etapa contemplará a elaboração, aplicação e análise de questionários para coleta de dados dos professores de Educação Física, Arte e coordenadores do ensino fundamental. Esses questionários proverão a fundamentação primordial para a elaboração dos artefatos Desplugados atingindo as necessidades e demandas elencadas pelos professores.

$\mathrm{Na}$ Terceira Etapa serão elaborados os artefatos Desplugados com base nas respostas obtidas pelos questionários da etapa anterior. Posteriormente essas atividades os artefatos gerados serão validados com os professores utilizando a sistemática aplicada por Kirkland et al. [2015]. A Quarta Etapa será compreendida pela elaboração do Guia de Pedagógico para Ensino/Prática de mindfulness por meio do PC, reunindo os artefatos Desplugados (Gibis) validados na etapa anterior e a sua aplicação pelos professores e/ou pelo pesquisador. Na Quinta Etapa, para a validação da pesquisa serão elaborados e 
VIII Congresso Brasileiro de Informática na Educação (CBIE 2019)

Anais dos Workshops do VIII Congresso Brasileiro de Informática na Educação (WCBIE 2019)

aplicados questionários que verifiquem se o desenvolvimento do $\mathrm{PC}$ e dos conceitos de mindfulness foram realmente desenvolvidos durante o processo.

\section{Resultados Esperados}

Acredita-se que a aplicação do Guia de Atividades Pedagógicas Desplugadas para ensino/prática de mindfulness aplicado às disciplinas de Educação Física e Arte amparadas pelos recursos metodológicos do PC Desplugado (Scratch) contribuem efetivamente ao estímulo da consciência corporal e espacial, criatividade e das habilidades estimuladas com o desenvolvimento dos pilares do PC e dos componentes do $\# 5$ c21 dos estudantes do ensino fundamental.

Também espera-se que o desenvolvimento do mindfulness por meio do PC Desplugado possa auxiliar na melhoria da atenção, e por consequente, no progresso do processo de ensino e aprendizagem dos estudantes de forma interdisciplinar em disciplinas diferentes das observadas por Santos [2019] na aplicação do PC Desplugado.

\section{Referências}

Ager, K., Albrecht, N., \& Cohen, M. (2015). Mindfulness in schools research project: Exploring students' perspectives of mindfulness-What are students' perspectives of learning mindfulness practices at school?. Psychology, 6(7), 896-914.

Alves, A. A. (2018). Melhorando a atenção do aluno através da tutoria de mindfulness: Um estudo de caso com o sistema tutor inteligente pat2math.

Black, D. S., \& Fernando, R. (2014). Mindfulness training and classroom behavior among lower-income and ethnic minority elementary school children. Journal of child and family studies, 23(7), 1242-1246.

Brackmann, C., Barone, D., Casali, A., Boucinha, R., \& Muñoz-Hernandez, S. (2016, September). Computational thinking: Panorama of the Americas. In 2016 international symposium on computers in Education (SIIE) (pp. 1-6). IEEE.

Brasil no PISA 2015 : análises e reflexões sobre o desempenho dos estudantes brasileiros / OCDE-Organização para a Cooperação e Desenvolvimento Econômico. - São Paulo : Fundação Santillana, 2016.

Burke, C. A. (2010). Mindfulness-based approaches with children and adolescents: A preliminary review of current research in an emergent field. Journal of child and family studies, 19(2), 133-144.

Gregory, G., \& Kaufeldt, M. (2015). The motivated brain: Improving student attention, engagement, and perseverance. ASCD.

Karunananda, A. S., Goldin, P. R., \& Talagala, P. D. (2016). Examining mindfulness in education. International Journal of Modern Education and Computer Science, 8(12), 23.

Kirkland, L. D., Manning, M., Osaki, K., \& Hicks, D. (2015). Increasing logico-mathematical thinking in low SES preschoolers. Journal of Research in Childhood Education, 29(3), 275-286.

Napoli, M., Krech, P. R., \& Holley, L. C. (2005). Mindfulness training for elementary school students: The attention academy. Journal of applied school psychology, 21(1), 99-125.

Rabiner, D. L., Murray, D. W., Skinner, A. T., \& Malone, P. S. (2010). A randomized trial of two promising computer-based interventions for students with attention difficulties. Journal of abnormal child psychology, 38(1), 131-142. 
VIII Congresso Brasileiro de Informática na Educação (CBIE 2019)

Anais dos Workshops do VIII Congresso Brasileiro de Informática na Educação (WCBIE 2019)

Ramos, D. K., de Melo, H. M., \& Mattar, J. (2018). Jogos digitais na escola e inclusão digital: intervenções para o aprimoramento da atenção e das condições de aprendizagem. Revista Diálogo Educacional, 18(58).

Rodrigues, S., Aranha, E., \& Silva, T. R. (2018, October). Computação Desplugada no Ensino de Programação: Uma Revisão Sistemática da Literatura. In Brazilian Symposium on Computers in Education (Simpósio Brasileiro de Informática na Educação-SBIE) (Vol. 29, No. 1, p. 417).

Romero, M. (2016). De l'apprentissage procédural de la programmation à l'intégration interdisciplinaire de la programmation créative. Formation et profession, 24(1), 87-89. https://doi.org/10.18162/fp.2016.a92

Romero,M.; Vallerand, V.; Nunes, M. A. S. N. (2019) Almanaque Para Popularização De Ciência Da Computação. Série 12: Guia Pedagógico; Volume 1: Atividades Tecnocriativas para crianças do século 21. ed. 1. Porto Alegre: SBC. v. 1. Disponível em: $\mathrm{http}: / /$ almanaquesdacomputacao.com.br/gutanunes/publications/S12V1.pdf

Santos, C. G. D. (2019). Estratégias para implantação e avaliação de um método educacional desplugado com histórias em quadrinhos para o ensino e aprendizagem associados ao desenvolvimento do pensamento computacional com alunos do ensino fundamental. Dissertação de mestrado, Universidade Federal de Sergipe, São Cristóvão, SE, Brasil.

Santos, C. G., Nunes M. A. S. N., Romero M. (2019) Guia de atividades desplugadas para o desenvolvimento do pensamento computacional. [] Porto Alegre, SBC, 201940 p.: il (Almanaque para a popularização de ciência da computação. Série 12, Guia pedagógico; v2) ISBN 978-85-7669-474-8

Simón, V. (2007). Mindfulness y neurobiología. Revista de psicoterapia, 66(67), 5-30.

Smith, C.; Strick, L. Dificuldades de aprendizagem de A a Z: guia completo para educadores e pais. Penso Editora, 2009.

Rahal, G. M. (2018). Mindfulness in the school context: Benefits and possibilities of integration. Psicologia Escolar e Educacional, 22(2), 347-358.

Williams, M., \& Penman, D. (2015). Atenção plena-Mindfulness: Como encontrar a paz em um mundo frenético. Sextante.

Wing, J. M. (2006). Computational thinking. Communications of the ACM, 49(3), 33-35.

Zenner, C., Herrnleben-Kurz, S., \& Walach, H. (2014). Mindfulness-based interventions in schools - a systematic review and meta-analysis. Frontiers in psychology, 5, 603. 